\title{
Pacific
}

Journal of

Mathematics

\section{CONFORMAL DEFORMATIONS PRESERVING THE GAUSS MAP}

\author{
ENALDO VERGASTA
}

Volume $156 \quad$ No. 2

December 1992 


\title{
CONFORMAL DEFORMATIONS PRESERVING THE GAUSS MAP
}

\author{
ENALdo Silva Vergasta
}

In this work, given a conformal immersion $f: M^{n} \rightarrow \mathbb{R}^{N}$ of a Riemannian manifold $M^{n}$ into a euclidean space $\mathbb{R}^{N}$, we establish conditions for the existence of another conformal immersion $\bar{f}: M^{n} \rightarrow$ $\mathbb{R}^{N}$ with the same Gauss map as $f$. In particular, for $n=2$ and $N=3$, these conditions are described by means of a partial differential equation on the principal curvatures of $f$.

0. Introduction. Let $M^{n}$ be a connected $n$-dimensional Riemannian manifold and let $f: M^{n} \rightarrow \mathbb{R}^{N}$ be a conformal immersion. We denote by $F: M^{n} \rightarrow G_{n, N}$ the Gauss map of $f$, which assigns to each point $p \in M^{n}$ the $n$-dimensional tangent space $f_{*}\left(T_{p} M\right)$ in the Grassmannian $G_{n, N}$. We consider the following problem: Under what conditions does there exist another conformal immersion $\bar{f}: M^{n} \rightarrow \mathbb{R}^{N}$ such that the Gauss map of $\bar{f}$ coincides with the Gauss map of $f$, up to a congruence in $G_{n, N}$ induced by a congruence in $\mathbb{R}^{N}$ ? When this occurs we say that $\bar{f}$ is a $G$-deformation of $f$. This situation is equivalent to considering conformal immersions $f$ and $\bar{f}$ with parallel tangent spaces $f_{*}\left(T_{p} M\right)$ and $\bar{f}_{*}\left(T_{p} M\right)$ in $\mathbb{R}^{N}$, which we will always assume. The analogous problem for isometric immersions $f$ and $\bar{f}$ was considered by Dajczer and Gromoll [D\&G].

In $\S 1$ we characterize our situation by means of a tensor field and a differentiable function satisfying certain conditions (see Proposition 1.5). This result will be used in $\S 2$, where we treat the above problem for $n=2$.

For surfaces, we also consider the oriented Gauss map $F^{*}: M^{2} \rightarrow$ $G_{2, N}^{*}$, where now $f_{*}\left(T_{p} M\right)$ is seen as an oriented 2-plane in the oriented Grassmannian $G_{2, N}^{*}$. In regard to the above problem we have two different situations. The first one is when $f$ and $\bar{f}$ have the same oriented Gauss map. In this case, it was shown by Hoffman and Osserman [H\&O-2] that either $f$ and $\bar{f}$ are minimal surfaces or $\bar{f}$ coincides with $f$ up to homothety and translation in $\mathbb{R}^{N}$. The other situation is when, for any local orientation in $M^{2}$, the oriented Gauss maps of $f$ and $\bar{f}$ differ by the orientation-reversing congruence in $G_{2, N}^{*}$. In this case we call $\bar{f}$ a $G^{*}$-deformation and say that 
$f$ is $G^{*}$-deformable. If $f$ is not totally umbilic, we show that a $G^{*}$ deformation is unique up to homothety and translation (Theorem 2.1). When $N=4$, we also prove that $G^{*}$-deformable immersions must have flat normal bundle. For $N=3$, Theorem 2.4 characterizes $G^{*}$ deformable immersions by means of a condition on their principal curvatures. We apply Theorem 2.4 to obtain $\bar{f}$ when $f$ is a rotation surface, a cyclid of Dupin or a surface with constant mean curvature. A similar result is obtained for constant mean curvature surfaces in the euclidean sphere $S^{3}$.

For hypersurfaces in $\mathbb{R}^{n+1}, n \geq 3$, the problem considered here will be treated in a forthcoming paper. Most of the results contained in these two works were announced in [Ve] and were obtained in my doctoral thesis. I wish to express my deep gratitude to Professor M. Dajczer for valuable advice and constant encouragement. I also thank the referee for many helpful suggestions.

1. Conformal deformation in $\mathbb{R}^{N}$ preserving the Gauss map. Let us denote by $\langle\cdot\rangle_{\circ}$ the Riemannian metric on $M^{n}$ and by $A_{\xi}$ the second fundamental form of the conformal immersion $f: M^{n} \rightarrow \mathbb{R}^{N}$ in the normal direction $\xi$, defined by

$$
f_{*} A_{\xi} X=-\left(\widetilde{\nabla}_{X} \xi\right)^{t},
$$

where ()$^{t}$ denotes the tangent projection along $f$ and $\tilde{\nabla}$ is the LeviCivita connection of the canonical metric $\langle$,$\rangle on the euclidean space$ $\mathbb{R}^{N}$. We denote also by $\langle$,$\rangle the metric on M^{n}$ induced by $f$, defined by $\langle\rangle=,e^{2 \varphi_{1}}\langle,\rangle_{0}$, where $e^{2 \varphi_{1}: M^{n}} \rightarrow \mathbb{R}$ is the conformal factor of $f$.

Let $\bar{f}: M^{n} \rightarrow \mathbb{R}^{N}$ be a $G$-deformation of $f$ with conformal factor $e^{2 \varphi_{2}}$. We define an orthogonal tensor field $T: T M \rightarrow T M$ by

$$
T=e^{-\varphi} f_{*}^{-1} \circ P \circ \bar{f}_{*},
$$

where $\varphi=\varphi_{2}-\varphi_{1}$ and, for each $q \in M^{n}$,

$$
P_{q}: T_{\bar{f}(q)} \mathbb{R}^{N} \rightarrow T_{f(q)} \mathbb{R}^{N}
$$

denotes the parallel transport in $\mathbb{R}^{N}$. For any vector field $V$ along $\bar{f}$ we have

$$
\widetilde{\nabla}_{X} P V=P \widetilde{\nabla}_{X} V
$$

where $X$ is any tangent field on $M^{n}$. We denote by $\nabla$ the LeviCivita connection on $M^{n}$ relative to the metric $\langle$,$\rangle and by \nabla \varphi$ the gradient of $\varphi$ with respect to this metric. The following result gives 
necessary and sufficient conditions on $T$ and $\varphi$ for the existence (at least locally) of a $G$-deformation of $f$.

Proposition 1.5. Let $f: M^{n} \rightarrow \mathbb{R}^{N}$ be a conformal immersion.

(i) If $\bar{f}$ is a $G$-deformation of $f$, then

$$
\nabla_{X} T=T \circ(X \wedge \nabla \varphi)
$$

and

$$
A_{\xi} \circ T=T^{-1} \circ A_{\xi}
$$

for any tangent field $X$ and normal field $\xi$. Moreover the second fundamental form $\bar{A}$ of $\bar{f}$ is given by

$$
\bar{A}_{\xi}=e^{-\varphi} T^{-1} \circ A_{P \xi} .
$$

(ii) If $M^{n}$ is simply connected and there exist an orthogonal tensor field $T$ and a differentiable function $\varphi$ satisfying (1.6) and (1.7), then for any $q_{0} \in M^{n}$,

$$
\bar{f}(q)=\int_{q_{0}}^{q} e^{\varphi} f_{*} T
$$

defines a G-deformation of $f$.

Proof. We will make use of the Gauss formula

$$
\widetilde{\nabla}_{X} f_{*} Y=f_{*} \nabla_{X} Y+\alpha(X, Y),
$$

where $X, Y \in T M$ and $\alpha(X, Y)$ denotes the normal component of $\widetilde{\nabla}_{X} f_{*} Y$. Recall the relationship between $\alpha$ and $A_{\xi}$, given by

$$
\langle\alpha(X, Y), \xi\rangle=\left\langle A_{\xi} X, Y\right\rangle \text {. }
$$

The Levi-Civita connection $\bar{\nabla}$ of the metric on $M^{n}$ induced by $\bar{f}$ (see [Ku], p. 316) is given by

$$
\bar{\nabla}_{X} Y=\nabla_{X} Y+X(\varphi) Y+Y(\varphi) X-\langle X, Y\rangle \nabla \varphi \text {. }
$$

Thus we can write

$$
\left(\widetilde{\nabla}_{X} \bar{f}_{*} Y\right)^{t}=\bar{f}_{*}\left(\nabla_{X} Y+X(\varphi) Y+Y(\varphi) X-\langle X, Y\rangle \nabla \varphi\right) .
$$

From (1.4), (1.9) and (1.10) we obtain

$$
\begin{aligned}
f_{*} \nabla_{X} T Y= & f_{*}\left(-X(\varphi) e^{-\varphi} f_{*}^{-1} P \bar{f}_{*} Y+e^{-\varphi} \nabla_{X} f_{*}^{-1} P \bar{f}_{*} Y\right) \\
= & -X(\varphi) e^{-\varphi} P \bar{f}_{*} Y+e^{-\varphi}\left(\widetilde{\nabla}_{X} P \bar{f}_{*} Y\right)^{t} \\
= & -X(\varphi) e^{-\varphi} P \bar{f}_{*} Y \\
& +e^{-\varphi} P \bar{f}_{*}\left(\nabla_{X} Y+X(\varphi) Y+Y(\varphi) X-\langle X, Y\rangle \nabla \varphi\right) \\
= & f_{*}\left(T \nabla_{X} Y+Y(\varphi) T X-\langle X, Y\rangle T \nabla \varphi\right),
\end{aligned}
$$

and this proves (1.6). 
If $\xi$ is a vector field normal to $f$, by $(1.1)$ and (1.4) we have

$$
\bar{f}_{*} \bar{A}_{\xi} X=-\left(\widetilde{\nabla}_{X} \xi\right)^{t}=-\left(P^{-1} \widetilde{\nabla}_{X} P \xi\right)^{t}=P^{-1} f_{*} A_{P \xi} X .
$$

Thus $\bar{A}_{\xi}=e^{-\varphi} T^{-1} A_{P \xi}$. Now (1.7) follows from the fact that $\bar{A}_{\xi}$ and $A_{P \xi}$ are self-adjoint.

In order to prove (ii), we compute the exterior differential of the 1 -form $e^{\varphi} f_{*} T$ defined on $M^{n}$ with values in $\mathbb{R}^{N}$ :

$$
\begin{aligned}
d\left(e^{\varphi} f_{*} T\right)(X, Y)= & \widetilde{\nabla}_{X} e^{\varphi} f_{*} T Y-\widetilde{\nabla}_{Y} e^{\varphi} f_{*} T X-e^{\varphi} f_{*} T([X, Y]) \\
= & X(\varphi) e^{\varphi} f_{*} T Y+e^{\varphi} \widetilde{\nabla}_{X} f_{*} T Y-Y(\varphi) e^{\varphi} f_{*} T X \\
& -e^{\varphi} \widetilde{\nabla}_{Y} f_{*} T X-e^{\varphi} f_{*} T\left(\nabla_{X} Y-\nabla_{Y} X\right) .
\end{aligned}
$$

Now we use (1.9) to get

$$
\begin{aligned}
d\left(e^{\varphi} f_{*} T\right)(X, Y)= & e^{\varphi}(\alpha(X, T Y)-\alpha(Y, T X)) \\
& +e^{\varphi} f_{*}\left(\nabla_{X} T Y-T \nabla_{X} Y-\nabla_{Y} T X+T \nabla_{Y} X\right. \\
& +X(\varphi) T Y-Y(\varphi) T X) .
\end{aligned}
$$

By (1.6) the above equality becomes

$$
d\left(e^{\varphi} f_{*} T\right)(X, Y)=e^{\varphi}(\alpha(X, T Y)-\alpha(Y, T X)) .
$$

But, for each vector $\xi$ normal to $f$ we have

$$
\begin{aligned}
& \langle\alpha(X, T Y)-\alpha(Y, T X), \xi\rangle \\
& \quad=\left\langle A_{\xi} X, T Y\right\rangle-\left\langle A_{\xi} T X, Y\right\rangle=\left\langle\left(T^{-1} A_{\xi}-A_{\xi} T\right) X, Y\right\rangle
\end{aligned}
$$

and this vanishes by (1.7). Thus $e^{\varphi} f_{*} T$ is a closed 1 -form on $M^{n}$. Since $M^{n}$ is simply connected, we can define $\bar{f}: M^{n} \rightarrow \mathbb{R}^{N}$ by

$$
\bar{f}(q)=\int_{q_{0}}^{q} e^{\varphi} f_{*} T .
$$

Then $\bar{f}_{*}=e^{\varphi} f_{*} T$ and $\left\langle\bar{f}_{*} X, \bar{f}_{*} Y\right\rangle=e^{2 \varphi}\langle X, Y\rangle$. So $\bar{f}$ is a $G$ deformation of $f$.

REMARK 1.11. As an immediate consequence of (1.6), we see that $\varphi$ is constant along $M^{n}$ if and only if $T$ is a parallel tensor field with respect to the metric $\langle$,$\rangle . When this occurs, f$ and $\bar{f}$ induce the same metric on $M^{n}$, up to a constant factor. Thus, in this ca्se the problem considered here is equivalent to considering isometric immersions $f$ and $\bar{f}$ with the same Gauss map. This was done by Dajczer and Gromoll in [D\&G], where the orthogonal tensor field (1.2) becomes $T=f_{*}^{-1} P \bar{f}_{*}$, is parallel and satisfies (1.7). 
2. Conformal deformations of surfaces preserving the Gauss map. In this section we study conformal surfaces in $\mathbb{R}^{N}$ that are $G^{*}$ deformable. In this case, the above tensor field $T$ must satisfy the additional condition $\operatorname{det} T=-1$ on $M^{2}$. We also obtain a result for surfaces with constant mean curvature in the euclidean sphere $S^{3}$. We begin with a uniqueness result.

THEOREM 2.1. Let $f: M^{2} \rightarrow \mathbb{R}^{N}$ be a conformal immersion which is not totally umbilic. If there exists a $G^{*}$-deformation $\bar{f}$, then $\bar{f}$ is unique up to homothety and translation in $\mathbb{R}^{N}$.

Proof. Let $\bar{M}^{2}$ denote $M^{2}$ with the opposite orientation. Denote the Gauss maps of $f: M^{2} \rightarrow \mathbb{R}^{N}$ and $\bar{f}: \bar{M}^{2} \rightarrow \mathbb{R}^{N}$ by $F$ and $\bar{F}$, respectively. Then $F=\bar{F}$ as maps of $M^{2}$ (without orientation) into $G_{2, N}^{*}$. Now apply Theorem 1.1 of [H\&O-1] and the basic uniqueness result in [H\&O-2].

Using some results of [We-1] and [We-2] we prove the following two theorems.

THEOREM 2.2. Let $f: M^{2} \rightarrow \mathbb{R}^{4}$ be a $G^{*}$-deformable conformal immersion. Then the normal bundle of $f$ is flat.

Proof. We may assume the Gauss map $F: M^{2} \rightarrow G_{2,4}^{*}$ is an immersion since the curvature of the normal bundle is zero anywhere $F$ fails to be regular. Then as in the proof of the previous theorem $F: M^{2} \rightarrow G_{2,4}^{*}$ and $\bar{F}: \bar{M}^{2} \rightarrow G_{2,4}^{*}$ are equal. Using Corollary 3 on p. 464 of [We-2], and the notation there, the existence of $f: M^{2} \rightarrow \mathbb{R}^{4}$ implies

$$
\varepsilon_{1}(g)+\rho_{1}(g)=\varepsilon_{2}(g)+\rho_{2}(g) ;
$$

from the existence of $\bar{f}: \bar{M}^{2} \rightarrow \mathbb{R}^{4}$, it follows that

$$
\varepsilon_{1}(g)-\rho_{1}(g)=\varepsilon_{2}(g)-\rho_{2}(g),
$$

where $g$ is the metric induced on $M^{2}$ by $f$. Thus $\varepsilon_{1}(g)=\varepsilon_{2}(g)$ and by Corollary 2 on p. 464 of [We-2] it follows that the normal bundle is flat.

THEOREM 2.3. Let $f: M^{2} \rightarrow \mathbb{R}^{N}$ with $N \geq 5$ be a conformal immersion. If there exists a point of $M^{2}$ which is not an inflection point of $f$, then $f$ is not $G^{*}$-deformable. 
Proof. This follows immediately from Proposition 5 of [We-1] and the observation that if $\bar{f}$ existed then as above $f: M^{2} \rightarrow \mathbb{R}^{N}$ and $\bar{f}: \bar{M}^{2} \rightarrow \mathbb{R}^{N}$ would have the same Gauss map.

The main result of this section is the following.

THEOREM 2.4. Let $f: M^{2} \rightarrow \mathbb{R}^{3}$ be a conformal immersion without umbilic points and let $a$ and $\omega$ be unit principal vector fields of $f$, with eigenvalues $\lambda$ and $\mu$ respectively.

(i) If $f$ is $G^{*}$-deformable, then

$$
(\lambda-\mu)(\alpha(\omega(\lambda))+\omega(\alpha(\mu)))+u(\mu) \omega(\mu)-u(\lambda) \omega(\lambda)=0 .
$$

(ii) If $M^{2}$ is simply connected and (2.5) is satisfied, then $f$ is $G^{*}$ deformable.

Proof. Let $p$ be any point $M^{2}$ and $\xi$ be a unit normal field defined on a neighborhood of $p$. Since $f$ has no umbilic points, there exist differentiable functions $\lambda$ and $\mu$, and orthonormal tangent fields $a$ and $w$, defined on a neighborhood of $p$, such that

$$
A_{\xi}=\lambda_{\omega}, \quad A_{\xi \omega}=\mu \omega .
$$

From Codazzi equation

$$
\nabla_{u}(\mu \omega)-A_{\xi} \nabla_{\omega} \omega=\nabla_{\omega}\left(\lambda_{\omega}\right)-A_{\xi} \nabla_{\omega} \iota
$$

we get

$$
\omega(\mu)=(\lambda-\mu)\left\langle\nabla_{\omega} u, \omega\right\rangle, \quad \omega(\lambda)=(\mu-\lambda)\left\langle\nabla_{u} \omega, u\right\rangle .
$$

Let us suppose that $f$ is $G^{*}$-deformable. Then there exist a differentiable function $\varphi: M^{2} \rightarrow \mathbb{R}$ and an orthogonal tensor field $T$ with $\operatorname{det} T=-1$, satisfying (1.6) and (1.7). Let $\alpha_{1}$ and $\omega_{1}$ be orthonormal tangent fields on a neighborhood of $p$ such that $T \iota_{1}=u_{1}$ and $T \omega_{1}=-\omega_{1}$. Then by (1.7) we have

$$
A_{\xi \iota_{1}}=A_{\xi} T^{-1} \iota_{1}=T A_{\xi \iota_{1}} \text {. }
$$

Thus $A_{\xi^{\iota_{1}}}$ is parallel to $\iota_{1}$. So $\iota_{1}$ and $\omega_{1}$ are principal directions, and this determines $T$ up to signal. From now on we will suppose that $T u=u$ and $T \omega=-\omega$. Now (1.6) is equivalent to

$$
u(\varphi)=-2\left\langle\nabla_{\omega} \iota, \omega\right\rangle, \quad \omega(\varphi)=-2\left\langle\nabla_{u} \omega, u\right\rangle .
$$

From (2.7) and (2.8) we have

$$
u(\varphi)=-\frac{2 u(\mu)}{\lambda-\mu}, \quad \omega(\varphi)=\frac{2 \omega(\lambda)}{\lambda-\mu} .
$$


By (2.8) we get

$$
\begin{aligned}
& {[\omega, \omega](\varphi)=\left(\nabla_{\omega} \omega\right)(\varphi)-\left(\nabla_{\omega} \omega\right)(\varphi)} \\
& =\left\langle\nabla_{a} \omega, \omega\right\rangle \alpha(\varphi)-\left\langle\nabla_{\omega} \omega, \omega\right\rangle \omega(\varphi) \\
& =0 \text {. }
\end{aligned}
$$

Thus we must have

$$
u(\omega(\varphi))-\omega(\alpha(\varphi))=0
$$

or, using (2.9),

$$
\omega\left(\frac{\omega(\lambda)}{\lambda-\mu}\right)+\omega\left(\frac{u(\mu)}{\lambda-\mu}\right)=0
$$

which is equivalent to $(2.5)$. Note that equation (2.5) is invariant by change of sign of the vector fields $\xi$, a and $\omega$. Thus it is valid everywhere on $M^{2}$.

Suppose now that $(2.5)$ is satisfied on $M^{2}$. We define the tangent vector field

$$
\delta=-2\left\langle\nabla_{\omega} u, \omega\right\rangle_{a}-2\left\langle\nabla_{u} \omega, u\right\rangle \omega
$$

and observe that $\delta$ does not depend on the unit vector fields $\xi$, $\alpha$ and $\omega$ satisfying (2.6). Now we define in $M^{2}$ the 1 -form $\gamma$ given by

$$
\gamma(X)=\langle\delta, X\rangle \text {. }
$$

Using (2.7) we compute

$$
\begin{aligned}
d \gamma(\alpha, \omega)= & \alpha(\gamma(\omega))-\omega(\gamma(\alpha))-\gamma\left(\nabla_{a} \omega-\nabla_{\omega} \alpha\right) \\
= & \frac{2}{\lambda-\mu}(\alpha(\omega(\lambda))+\omega(\mu(\mu))) \\
& +\frac{2}{(\lambda-\mu)^{2}}(\alpha(\mu) \omega(\mu)-\alpha(\lambda) \omega(\lambda)) .
\end{aligned}
$$

By (2.5) the 1-form $\gamma$ is closed. Since $M^{2}$ is simply connected, there exists $\varphi: M^{2} \rightarrow \mathbb{R}$ such that $\nabla \varphi=\delta$, that is,

$$
\omega(\varphi)=-2\left\langle\nabla_{\omega} \omega, \omega\right\rangle, \quad \omega(\varphi)=-2\left\langle\nabla_{u} \omega, u\right\rangle .
$$

We define the tensor field $T$ by $T \omega=\omega$ and $T \omega=-\omega$. Then $T$ is orthogonal, det $T=-1$ and (1.7) is satisfied. From (2.12) it is easy to show that (1.6) is satisfied. By Proposition 1.5, $f$ is $G^{*}$ deformable. 
The next result is needed for the proof of some of the corollaries to Theorem 2.4.

Proposition 2.13. If $(x, y)$ are principal coordinates on an open set $U \subset M^{2}$, then on $U(2.5)$ is equivalent to

$$
\left(\frac{(\lambda+\mu)_{x}}{\lambda-\mu}\right)_{y}+\left(\frac{(\lambda+\mu)_{y}}{\lambda-\mu}\right)_{x}=0 .
$$

Proof. Let $E$ and $G$ be positive functions such that

$$
\frac{\partial}{\partial x}=E_{\iota} \quad \text { and } \quad \frac{\partial}{\partial x}=G \omega .
$$

Since $\left[\frac{\partial}{\partial x}, \frac{\partial}{\partial y}\right]=0$ we obtain

$$
\begin{aligned}
E_{u}(G)-E G\left\langle\nabla_{\omega \iota}, \omega\right\rangle & =0, \\
-G \omega(E)+E G\left\langle\nabla_{u} \omega, \iota\right\rangle & =0,
\end{aligned}
$$

and then by (2.7) we have

$$
G_{x}=\frac{G \mu_{x}}{\lambda-\mu}, \quad E_{y}=-\frac{E \lambda_{y}}{\lambda-\mu} .
$$

From (2.15) and (2.16) it follows that

$$
\begin{aligned}
& \omega(\mu(\mu))=\frac{\mu_{x y}}{E G}+\frac{\mu_{x} \lambda_{y}}{(\lambda-\mu) E G}, \\
& u(\omega(\lambda))=\frac{\lambda_{x y}}{E G}-\frac{\mu_{x} \lambda_{y}}{(\lambda-\mu) E G},
\end{aligned}
$$

and then (2.5) and (2.14) are equivalent.

In the next two corollaries, we will consider $f$ as the inclusion map of an open subset of $M^{2}$ in $\mathbb{R}^{3}$.

COROLlaRY 2.17. Let $M^{2}$ be a rotation surface which does not meet its axis of symmetry. Then $f$ is $G^{*}$-deformable. If $M^{2}$ is not totally umbilic then $\bar{f}$ is unique up to homothety and translation; also, $\bar{f}(M)$ is again a rotation surface. If $M^{2}$ is part of a sphere and $\bar{f}(M)$ is a rotation surface, then $\bar{f}(M)$ is part of a catenoid.

Proof. We take on $f\left(M^{2}\right)$ the parametrization $\phi:(0,2 \pi) \times I \rightarrow \mathbb{R}^{3}$ given by

$$
\phi(x, y)=(\alpha(y) \cos x, \alpha(y) \sin x, \beta(y)),
$$


where $(\alpha(y), \beta(y))$ is a plane curve defined on an open interval $I \subset \mathbb{R}$ and satisfying $\alpha(y) \neq 0$ for any $y \in I$.

Now define $\varphi: M^{2} \rightarrow \mathbb{R}$ by $e^{\varphi}=1 / \alpha^{2}(y)$. One can immediately verify that

$$
\bar{\phi}(x, y)=\left(\frac{1}{\alpha(y)} \cos x, \frac{1}{\alpha(y)} \sin x, \bar{\beta}(y)\right),
$$

where

$$
\bar{\beta}(y)=\int_{y_{0}}^{y} \frac{\beta^{\prime}(t)}{\alpha^{2}(y)} d t,
$$

satisfy $\bar{\phi}_{x}=e^{\varphi} \phi_{x}$ and $\bar{\phi}_{y}=-e^{\varphi(y)} \phi_{y}$. Thus $\bar{f}$ defined by $\bar{f}(\phi(x, y)$ $=\bar{\phi}(x, y)$ is a $G^{*}$-deformation. Now, if $M^{2}$ is part of a sphere, then up to homothety and translation $f$ is the normal Gauss map of $f$ into the unit sphere $S^{2}$. So $\bar{f}\left(M^{2}\right)$ must be a minimal surface, hence it is part of a catenoid.

A surface in $\mathbb{R}^{3}$ that is the envelope of a family of spheres tangent to three fixed spheres in $\mathbb{R}^{3}$ is called a cyclid of Dupin. These surfaces can be characterized by the fact that they are the surfaces without umbilic points whose principal curvatures are constant along the respective curvature lines (see [C\&R], pp. 151-166).

COROLlary 2.19. Let $M^{2}$ be a cyclid of Dupin and $U$ an open simply connected subset of $M^{2}$. Then $f$ restricted to $U$ is $G^{*}$-deformable.

Proof. If $(x, y)$ are principal coordinates, then the respective principal curvatures $\lambda$ and $\mu$ satisfy $\lambda_{x}=\mu_{y}=0$. Thus (2.14) is verified and we can apply Theorem 2.4 .

REMARK 2.20. In the preceding corollary, we have by (2.9),

$$
\varphi=\log \left(c(\lambda-\mu)^{2}\right)
$$

for some positive constant $c \in \mathbb{R}$. By (1.8), the principal curvatures of $\bar{f}$ are

$$
\bar{\lambda}=\frac{c \lambda}{(\lambda-\mu)^{2}}, \quad \bar{\mu}=\frac{-c \mu}{(\lambda-\mu)^{2}} .
$$

Thus, in general, the new surface $\bar{f}(U)$ is not a cyclid of Dupin.

Corollary 2.21. Let $f: M^{2} \rightarrow \mathbb{R}^{3}$ be an oriented minimal surface without umbilic points and let $N: M^{2} \rightarrow S^{2} \subset \mathbb{R}^{3}$ be the normal Gauss map. Then $f$ is $G^{*}$-deformable and $\bar{f}=N$ up to homothety and translation. 
Proof. Taking principal coordinates $(x, y)$, we have

$$
N_{x}=-\lambda \frac{\partial}{\partial x}, \quad N_{y}=\lambda \frac{\partial}{\partial y} \text {. }
$$

Thus the corollary is a consequence of Theorem 2.1 .

COROLlARY 2.22. Let $f: M^{2} \rightarrow \mathbb{R}^{3}$ be an oriented surface free of umbilic points, with constant mean curvature $H \neq 0$, and let $N: M^{2} \rightarrow$ $S^{2} \subset \mathbb{R}^{3}$ be the normal Gauss map. Then $f$ is $G^{*}$-deformable and $\bar{f}$ is the parallel surface $g=f+\frac{1}{H} N$, up to homothety and translation.

Proof. Taking principal coordinates $(x, y)$, we have

$$
g_{x}=f_{x}+\frac{1}{H} N_{x}=\left(1-\frac{\lambda}{H}\right) f_{x}=\left(\frac{\mu-\lambda}{\lambda+\mu}\right) f_{x}
$$

and

$$
g_{y}=\left(\frac{\lambda-\mu}{\lambda+\mu}\right) f_{y} .
$$

Thus $g$ is a $G^{*}$-deformation of $f$. We observe that, since

$$
N_{x}=-\lambda f_{x}=\lambda\left(\frac{\lambda+\mu}{\lambda-\mu}\right) \bar{f}_{x}
$$

and

$$
N_{y}=-\mu f_{y}=-\mu\left(\frac{\lambda+\mu}{\lambda-\mu}\right) \bar{f}_{y},
$$

one sees that the mean curvature of $\bar{f}$ is also $H$.

We conclude this work with a result analogous to the preceding corollary, for a constant mean curvature surface in $S^{3}$.

Proposition 2.23. Let $f: M^{2} \rightarrow S^{3}$ be an oriented surface free of umbilic points, with constant mean curvature $H$, and let $N: M^{2} \rightarrow S^{3}$ be a vector field normal to $f$. Then $f$ (seen as a surface in $\mathbb{R}^{4}$ ) is $G^{*}$-deformable and $\bar{f}$ is the parallel surface

$$
\bar{f}=\frac{1}{\left(H^{2}+1\right)^{1 / 2}}(N+H f): M^{2} \rightarrow S^{3},
$$

up to homothety and translation.

Proof. It is analogous to the preceding proof.

REMARK 2.24 . One can easily check that the above immersion $\bar{f}$ has constant mean curvature in $S^{3}$. When $H=0,-\bar{f}$ is the polar map of the minimal immersion $f$, as defined by Lawson [La]. 


\section{REFERENCES}

[C\&R] T. E. Cecil, and P. J. Ryan, Tight and taut immersions of manifolds, Research Notes in Math., Pitman Adv. Pub. Prog., 1985.

[D\&G] M. Dajczer and D. Gromoll, Real Kaehler submanifolds and uniqueness of the Gauss map, J. Differential Geom., 22 (1985), 13-28.

[H\&O-1] D. Hoffman and R. Osserman, The geometry of the generalized Gauss map, Mem. Amer. Math. Soc., no. 236, (1980).

[H\&O-2] - The Gauss map of surfaces in $\mathbb{R}^{n}$, J. Differential Geom., 19 (1982), 733-754.

[Ku] R. S. Kulkarni, Curvature and metric, Ann. of Math., 91 (1970), 311-333.

[La] H. B. Lawson, Jr., Complete minimal surfaces in $S^{3}$, Ann. of Math., 92 (1970), 335-374.

[Ve] E. S. Vergasta, Conformal immersions with the same Gauss map, An. Acad. Brasil Ci., 59 (1987), 145-147.

[We-1] J. L. Weiner, The Gauss map for surfaces: Part 1-The affine case, Trans. Amer. Math. Soc., 293 (1986), 431-446.

[We-2] _ The Gauss map for surfaces: Part 2-The Euclidean case, Trans. Amer. Math. Soc., 293 (1986), 447-466.

Received May 2, 1988 and in revised form August 25, 1991.

UNIVERSIDAde FEDERAL dA BAHIA

CEP 40210, SAlVAdor, BA, BrazIL 



\title{
PACIFIC JOURNAL OF MATHEMATICS \\ Founded by \\ E. F. BeCKenbach (1906-1982) F. Wolf (1904-1989)
}

\section{EDITORS}

\author{
V. S. VARADARAJAN \\ (Managing Editor) \\ University of California \\ Los Angeles, CA 90024-1555 \\ vsv@math.ucla.edu \\ Herbert Clemens \\ University of Utah \\ Salt Lake City, UT 84112 \\ clemens@math.utah.edu \\ F. Michael Christ \\ University of California \\ Los Angeles, CA 90024-1555 \\ christ@math.ucla.edu \\ THOMAS ENRIGHT \\ University of California, San Diego \\ La Jolla, CA 92093 \\ tenright@ucsd.edu
}

\author{
Nicholas ERcolani \\ University of Arizona \\ Tucson, AZ 85721 \\ ercolani@math.arizona.edu \\ R. FINN \\ Stanford University \\ Stanford, CA 94305 \\ finn@gauss.stanford.edu \\ VAughan F. R. Jones \\ University of California \\ Berkeley, CA 94720 \\ vfr@math.berkeley.edu \\ STEVEN KeRCKHOFF \\ Stanford University \\ Stanford, CA 94305 \\ spk@gauss.stanford.edu
}

\author{
C. C. MOORE \\ University of California \\ Berkeley, CA 94720
}

MARTIN SCHARLEMANN

University of California

Santa Barbara, CA 93106

mgscharl@henri.ucsb.edu

\author{
HAROLD STARK \\ University of California, San Diego \\ La Jolla, CA 92093
}

\section{SUPPORTING INSTITUTIONS}

UNIVERSITY OF ARIZONA
UNIVERSITY OF BRITISH COLUMBIA
CALIFORNIA INSTITUTE OF TECHNOLOGY
UNIVERSITY OF CALIFORNIA
MONTANA STATE UNIVERSITY
UNIVERSITY OF NEVADA, RENO
NEW MEXICO STATE UNIVERSITY
OREGON STATE UNIVERSITY

\author{
UNIVERSITY OF OREGON \\ UNIVERSITY OF SOUTHERN CALIFORNIA \\ STANFORD UNIVERSITY \\ UNIVERSITY OF HAWAII \\ UNIVERSITY OF TOKYO \\ UNIVERSITY OF UTAH \\ WASHINGTON STATE UNIVERSITY \\ UNIVERSITY OF WASHINGTON
}

The Supporting Institutions listed above contribute to the cost of publication of this Journal, but they are not owners or publishers and have no responsibility for its content or policies.

\begin{abstract}
Mathematical papers intended for publication in the Pacific Journal of Mathematics should be in typed form or offset-reproduced (not dittoed), double spaced with large margins. Please do not use built up fractions in the text of the manuscript. However, you may use them in the displayed equations. Underline Greek letters in red, German in green, and script in blue. The first paragraph must be capable of being used separately as a synopsis of the entire paper. In particular it should contain no bibliographic references. Please propose a heading for the odd numbered pages of less than 35 characters. Manuscripts, in triplicate, may be sent to any one of the editors. Please classify according to the 1991 Mathematics Subject Classification scheme which can be found in the December index volumes of Mathematical Reviews. Supply name and address of author to whom proofs should be sent. All other communications should be addressed to the managing editor, or Elaine Barth, University of California, Los Angeles, California 90024-1555.

There are page-charges associated with articles appearing in the Pacific Journal of Mathematics. These charges are expected to be paid by the author's University, Government Agency or Company. If the author or authors do not have access to such Institutional support these charges are waived. Single authors will receive 50 free reprints; joint authors will receive a total of 100 free reprints. Additional copies may be obtained at cost in multiples of 50 .
\end{abstract}

The Pacific Journal of Mathematics (ISSN 0030-8730) is published monthly except for July and August. Regular subscription rate: $\$ 190.00$ a year (10 issues). Special rate: $\$ 95.00$ a year to individual members of supporting institutions.

Subscriptions, orders for numbers issued in the last three calendar years, and changes of address should be sent to Pacific Journal of Mathematics, P.O. Box 969, Carmel Valley, CA 93924, U.S.A. Old back numbers obtainable from Kraus Periodicals Co., Route 100, Millwood, NY 10546.

The Pacific Journal of Mathematics at P.O. Box 969, Carmel Valley, CA 93924 (ISSN 0030-8730) is published monthly except for July and August. Second-class postage paid at Carmel Valley, California 93924, and additional mailing offices. Postmaster: send address changes to Pacific Journal of Mathematics, P.O. Box 969, Carmel Valley, CA 93924.

\section{PUBLISHED BY PACIFIC JOURNAL OF MATHEMATICS, A NON-PROFIT CORPORATION} Copyright (C) 1992 by Pacific Journal of Mathematics 


\section{PACIFIC JOURNAL OF MATHEMATICS}

Volume $156 \quad$ No. $2 \quad$ December 1992

Surfaces in the 3-dimensional Lorentz-Minkowski space satisfying 201 $\Delta x=A x+B$

Luis Alías, Angel FerRandez and Pascual LuCAS

Lie algebras of type $D_{4}$ over number fields

209

BRUCE ALLISON

Subsemigroups of completely simple semigroups

251

Anne Antonippillai and Francis PAStiJn

Studying links via closed braids. VI. A nonfiniteness theorem

JOAN Birman and William W. MENASCO

Minimal orbits at infinity in homogeneous spaces of nonpositive curvature

MARÍA J. DRUETTA

Generalized horseshoe maps and inverse limits

SARAH ElizABETH Holte

Determinantal criteria for transversality of morphisms

DAN LAKSOV and ROBERT SPEISER

Four dodecahedral spaces

PETER LORIMER

Semifree actions on spheres

MONICA NiCOLAU

Conformal deformations preserving the Gauss map

ENALDO VERGASTA

Hecke eigenforms and representation numbers of arbitrary rank lattices 371

LYNNE WALLING 